\title{
Research on the Suitability Design of University Gate
}

\author{
Liang Haixiu ${ }^{1}$, Fan Ziyi ${ }^{1, *}$ \\ ${ }^{1}$ School of Architecture, South China University of Technology, Guangzhou, Guangdong, China
}

\begin{abstract}
With the development of university campus construction, the campus planning and architectural design concept also presents a variety of characteristics. The university gate is an important part of the university campus city interface, which is of great significance for the display of the overall image of the campus and the education concept. On the basis of summarizing the development process and morphological characteristics of university gate, this paper analyzes the suitability of its scale, culture and landscape, and puts forward corresponding design strategies. The paper concludes that the school gate scale suitability design should be based on people, from the perspective of human comfort, to determine the appropriate scale, cultural suitability design should take into account the discipline characteristics and regional culture, landscape suitability design should adapt to the climate characteristics.
\end{abstract}

\section{Introduction}

Universities, also known as institutions of higher learning, are places for students and teachers to study and live. The quality of campus environment is closely related to the growth of students and the development of knowledge.

In recent years, with the development and renewal of the concept of higher education, the construction of university campus also has an unprecedented development, from the previous extensive development model to the development model focusing on the quality of campus space. The gate of a university is not only the connection channel between the campus space and the outside world, but also the display window of the university to the outside world ${ }^{[1]}$. The development of university campus also brings the development of university gate design.

This paper intends to summarize the development process and form types of university gate, focusing on the analysis of the influencing factors of the scale, culture and landscape suitability design of university gate, and puts forward the corresponding design methods and suggestions, hoping to provide some reference for the future university gate design.

\section{Overview of the development of university gate}

Chinese higher education research institutions have a long history, which can be traced back to the academies sprouting from the Tang Dynasty. Most of the academies adopt symmetrical layout and courtyard style combination. Different courtyard spaces correspond to different functions of the academies, with clear zoning, forming a relatively strict architectural group. The gate of the academy is generally set at the end of the central axis of the building group, and some of them also have multiple gates. The gate can be divided into two types: the house type and the archway type. The building type gate is mainly in the form of three hard mountain buildings, such as Yuelu Academy (Fig. 1); The archway gate is mainly in the form of three rooms and four columns, such as Wuyi Academy (Fig. 2).

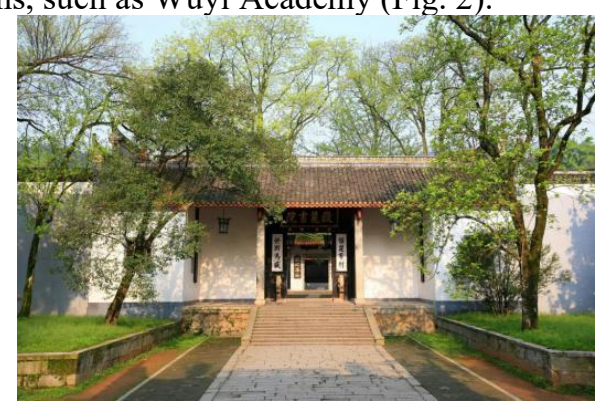

Fig. 1. Yuelu Academy

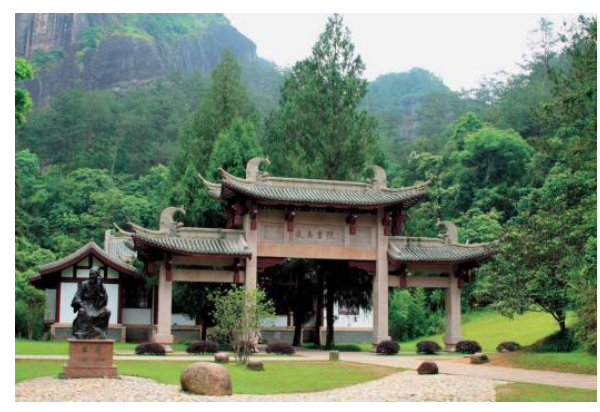

Fig. 2. Wuyi Academy

At the end of the 19th century, in the period of Westernization Movement, westernization school was established in China. During this period, the school planning and school gate style were largely influenced by the west, most of which adopted the western classical

\footnotetext{
$\overline{{ }^{*} \text { Corresponding author: } 1185898962 @ q q . c o m}$
} 
form, while some national school gates adopted the traditional Chinese archway form. In the early days of the founding of the people's Republic of China, the construction mode of colleges and universities was similar to that of the Soviet Union, and the school gate style was similar to that of the Soviet Union; After the reform and opening up, due to the change of concept and the development of education concept, great changes have taken place in the planning and gate design of the University. The gate design of the university has become more concerned about the overall landscape and environment of the campus, reflecting the diversity.

It can be seen that the development of University gates in China is a changing process from "tradition" to "modern" and from "singleness" to "diversity".

\section{Research on the suitability design of university gate}

\subsection{The suitability of university gate scale}

\subsubsection{Analysis on the scale of university gate}

As the window of the University, the school gate often affects the first impression of the society on the University. However, due to the pursuit of the lofty image of the University, some universities lead to the phenomenon of the scale imbalance of the University ${ }^{[2]}$. On the contrary, it has a negative impact on the image of the university to a certain extent. In fact, it brings people depressed and uncomfortable psychological feelings. Therefore, it is particularly urgent to study the scale suitability of university gate.

\subsubsection{Sense of visual scale}

Scale is a standard to measure things, among which "ruler" is the size, the measurement unit of the size and length of the object, and "degree" is a measure and a degree, and it is the relative size of the quantity produced by the comparison of the relationship between quantity and quantity ${ }^{[3]}$. Although the concept of scale in the context of architecture has something to do with the real size of objects, it is essentially an expression of people's feelings about the relationship between the size of space. Because visual experience is the most direct way for people to experience space, visual sense of scale is the main factor to determine the scale.

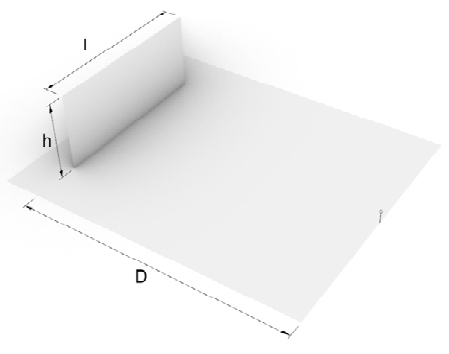

Fig. 3. Model diagram

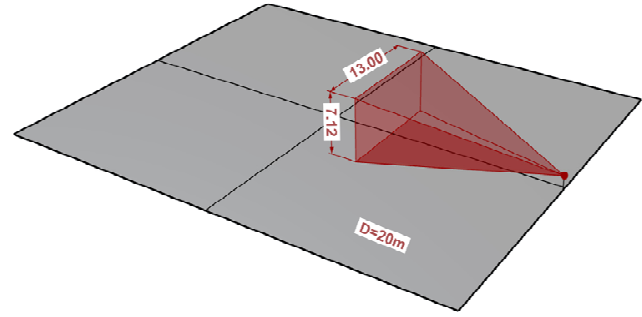

Fig. 4. Calculation results when $D=20 \mathrm{~m}$

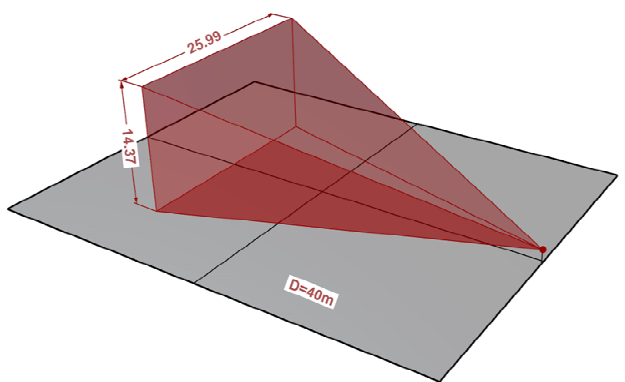

Fig. 5. Calculation results when $D=40 \mathrm{~m}$

The sense of visual scale is related to human visual comfort. According to the research on human visual comfort, human visual angle is sensitive to dynamic things at 20-30 degrees. When the horizontal angle of view is 36 degrees and the vertical angle of view is 20 degrees, the human eye can have a better visual experience, and the scope of the object can make the observer easily understand. Therefore, based on the perspective of comfort, we can calculate the appropriate size of the university gate. By controlling the value of independent variable $\mathrm{D}$, the size of the school gate at a certain observation distance can be obtained under the condition of 36 degrees in the horizontal direction and 20 degrees in the vertical direction, that is, the value of dependent variables $h$ and $L$. Combined with the actual situation, the distance $\mathrm{d}$ from the observation point to the school gate should be determined according to the maximum distance from the construction location of the school gate to the side line of the square. The size of the school gate obtained from this is the maximum value in line with the visual comfort, and the size of the school gate based on the visual comfort should not be greater than this value (Table 1).

Table 1. Numerical table of university gate dimensions based on visual comfort

\begin{tabular}{|l|l|l|}
\hline $\begin{array}{c}\text { Distance from } \\
\text { observation point to } \\
\text { school gate }(\mathrm{m})\end{array}$ & $\begin{array}{c}\text { The height } \\
\text { of gate } \\
(\mathrm{m})\end{array}$ & $\begin{array}{c}\text { The width of } \\
\text { gate }(\mathrm{m})\end{array}$ \\
\hline 15 & 5.32 & 9.75 \\
\hline 20 & 7.12 & 13.00 \\
\hline 25 & 8.93 & 16.25 \\
\hline 30 & 10.74 & 19.50 \\
\hline 40 & 14.37 & 25.99 \\
\hline
\end{tabular}




\subsection{Cultural suitability of university gate}

\subsubsection{The influence factors of university gate culture}

\section{(1) Subject characteristics}

With the development of the University, the cultural needs of the university campus have become increasingly prominent ${ }^{[2]}$. The school gate has become one of the symbols of the university campus culture, reflecting the cultural heritage and image characteristics of the University. According to the different disciplines, Chinese universities can be divided into comprehensive, science and engineering, normal, agriculture and forestry, medicine and other colleges. The campus culture of different types of colleges can be reflected from the characteristics of the school gate, and can be used as the propaganda point of the campus. In reality, the construction of the school gate shows the characteristics of assimilation, which makes it difficult to reflect the disciplinary characteristics of the campus.

(2) Regional culture

Architectural design needs to adapt to the local climate characteristics, topography, surrounding environment, while continuing the local cultural characteristics, in order to take root in the region. University campus is located in different regions, and its unique regional culture can be reflected through the architectural style and form of the campus, integrating into the local environment.

\subsubsection{Cultural suitability design strategy}

According to the investigation of different types of University gates, we can summarize the different forms of campus culture. Comprehensive colleges generally have a long history and profound cultural heritage. The school gates tend to adopt the classical and Chinese style archways $^{[6]}$. For example, the main school gate of Peking University and the school gate of Nanjing University all adopt the classical archway style (Figure 6). For example, the southeast gate of Tsinghua University is made of a whole block of stone, which is engraved with the name and motto of the school and integrated with the surrounding environment (Figure 7). Normal colleges and universities pay attention to normal education, and their school style is mainly solemn, which reflects the moral standard of being upright and learning as a teacher. Medical colleges and universities generally adopt a simple form. Some of them will set medical sculptures at the school gate. For example, in the gate design of Guangdong University of traditional Chinese medicine, books and sculptures of Huangdi Neijing and Shennong materia medica are set on both sides of the school gate, which clearly reflects the school's traditional Chinese medicine culture.

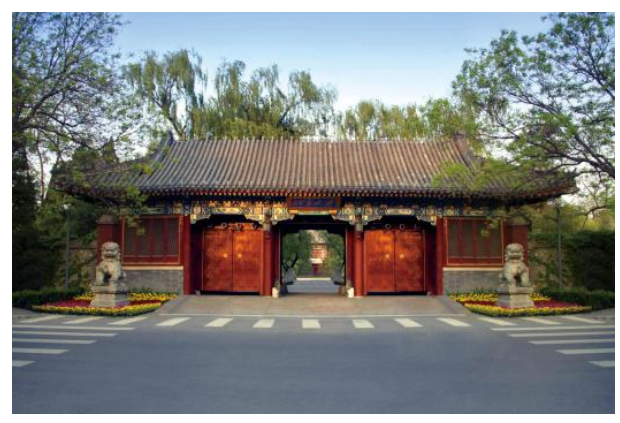

Fig. 6. The gate of Peking University

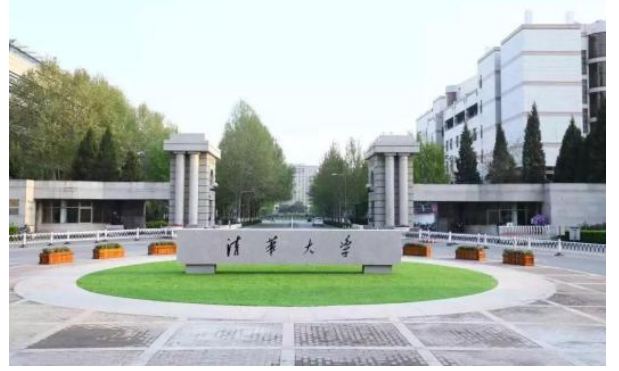

Fig. 7. The gate of Tsinghua University

Considering the local cultural background, the university gate design should reflect the regional characteristics $^{[4]}$. For example, the gate of Wuhan University is a classical archway gate with blue glazed tiles, which is consistent with the main color of Luojia mountain, making the gate square and the surrounding natural environment complement each other, achieving a perfect integration with the environment (Figure 8). The east gate of Yantai University abstracts and deforms the image elements related to the sea, such as sails, waves, masts, etc., and transforms them into various elements of the gate, which are skillfully combined together. The overall image is just like a sailing ship, giving people the imagination of strength and beauty, while showing the distinctive coastal architectural characteristics (Figure 9).

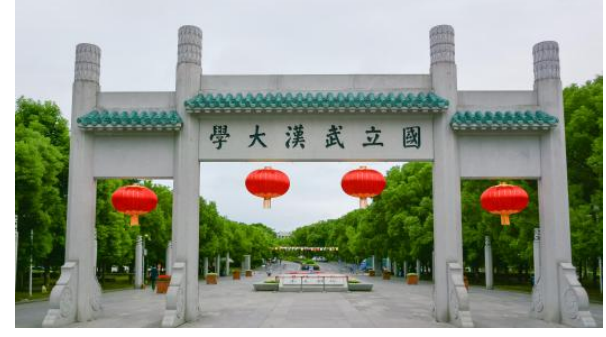

Fig. 8. The gate of Wuhan University

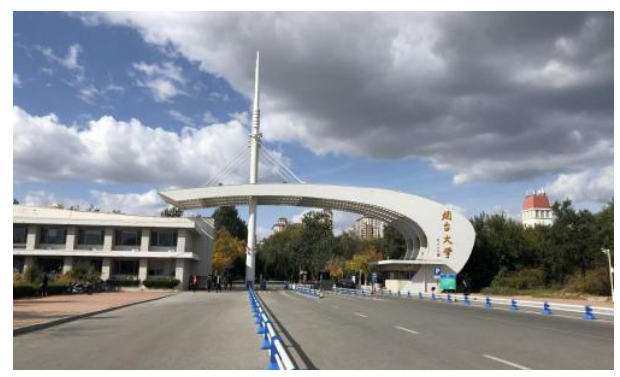

Fig.9. The gate of Yantai University 


\subsection{Landscape suitability of university gate}

\subsubsection{Influencing factor of landscape design of university gate}

Climate is the annual average state of the atmosphere in a region. The main climate elements include light, temperature and precipitation. Different climate characteristics will affect the growth and type distribution of plants, thus forming different landscapes ${ }^{[5]}$.

\subsubsection{Landscape suitability design strategy}

The planning and design of school gate landscape needs to consider the selection of local tree species, pay attention to the needs of the main users of the campus for the comfort of the campus environment, and improve the use efficiency of the campus public space. For example, in the subtropical monsoon climate region, the summer is hot and the winter is warm, the four seasons are distinct, and the precipitation is abundant, but the monsoon characteristics are obvious, and the tree species are mostly subtropical evergreen broad-leaved forest. Therefore, when carrying out landscape design in this region, we should pay attention to the experience of space under the tree, and tend to choose broad-leaved forest trees to provide more shade space. We should pay attention to the reasonable collocation of trees, shrubs and lawns in the greening configuration, so as to form an orderly landscape level. We should also pay attention to the collocation of tree species in different flowering periods, so as to form different scenery in four seasons.

In order to meet the actual needs of a large number of visitors and group photo taking, the school gate should be set with a hard square, and be properly separated from the motor vehicle lane to ensure the separation of people and vehicles in the square. The open space of the school gate is generally set in the green space on both sides of the school gate or in the middle. The parking space of the school gate is divided into motor vehicle parking and non motor vehicle parking, usually combined with the green space on both sides of the school gate.

\section{Conclusion}

Suitability design is of great significance in the planning and design of University gates. It can not only better show the overall image of the university, but also greatly improve the quality of campus public space. Therefore, architectural designers should really start from the user's point of view, people-oriented, make full use of the local regional cultural advantages, and create a modern university gate that not only has regional cultural characteristics, but also conforms to the aesthetic style of the new era. This paper summarizes the development process and morphological characteristics of the university gate, then analyzes the suitability of the gate's scale, culture and landscape, and puts forward the corresponding design strategies. It is concluded that the gate's scale suitability design should be people-oriented, from the perspective of human comfort, and the cultural suitability design should take into account the discipline characteristics and regional culture, Landscape suitability design should adapt to climate characteristics. In order to adapt to the development of the times, the suitability design of university gate is not limited to the functions of on duty management. In addition, it can be studied from the perspective of rational and economical university gate design ${ }^{[8]}$.

\section{References}

1. D. Lu, Y. Zhang, Urban architecture, 16 (03), 49-51 +85 (2019)

2. X. Wang, Shanxi architecture,40 (04),31-33 (2014)

3. D. Wang, C. Huang, Sichuan architectural science research, 04, 100-102(2003)

4. F. Pan, Shenzhen University, Research on gate design of universities in Guangzhou, Shenzhen and Hong Kong (2019)

5. N. Li, Xi'an University of architecture and technology, Investigation and analysis of campus entrance space (2008)

6. T. Wen, B. Qiu, Architecture and culture,02,110111(2012)

7. B. Luo, Kunming University of science and technology, Landscape analysis of campus entrance space (2011)

8. W. Yin, Hefei University of technology, Investigation and analysis of Chinese universities (2007) 